Bull. Austral. Math. Soc.

$34 \mathrm{~B} 10,35 \mathrm{~K} 50,54 \mathrm{H} 20,80 \mathrm{~A} 25$

VoL. 66 (2002) [393-403]

\title{
CHAPMAN-JOUGUET DETONATION PROFILE FOR A QUALITATIVE MODEL
}

\begin{abstract}
ABDolRahman RAZANI
In this article, the existence of traveling wave fronts for a one step chemical reaction with a natural discontinuous reaction rate function is studied. This discontinuity occurs because of the cold boundary difficulty and implies a discontinuous system of ordinary differential equations. By some general topological arguments in ordinary differential equations, the Chapman-Jouguet detonation for exothermic reactions is shown to exist. In addition, the uniqueness of this wave is considered.
\end{abstract}

\section{INTRODUCTION}

The Chapman-Jouguet detonation wave problem for a compressible reacting gas, with one reactant involved in a single step chemical reaction is studied. For mathematical reasons we can only handle the case of exothermic, that is, irreversible reactions. Majda [6] develops a simpler asymptotic model system, valid in the "Mach $1+\varepsilon$ " regime, which still incorporates substantial interaction of chemical and fluid mechanical phenomena, but has solutions which are easier to analyse.

The model describes the detailed structure and the propagation of a reacting gas front into a combustible mixture and is as follows:

$$
\begin{gathered}
\frac{d}{d \tau} T+\{f(T)\}_{x}-q_{0} Z_{x}=\beta T_{x x} \\
Z_{x}=\kappa \Phi(T) Z
\end{gathered}
$$

where $\kappa>0$ is a constant, $T$ is the temperature, $Z$ in the mass fraction of unburned gas (note that the completely unburned gas corresponds to $Z=1$ and a totally burned gas corresponds to $Z=0$ ), and $\tau$ is a variable proportional to the arc length along the characteristics. The coordinate $x$ is not the space coordinate but is determined through the asymptotics as a scaled space-time coordinate representing distance to the reaction zone (the $x$-differentiation occurs because $Z$ in (1.1) is convected at the much slower fluid velocity rather than the much faster reacting shock speed (see [1] or [7] for details). Finally $q_{0}>0$ is the effective heat release from the chemical reaction, and

Received 26th February, 2002

Copyright Clearance Centre, Inc. Serial-fee code: 0004-9727/02 \$A2.00+0.00. 
$\beta>0$ is a lumped viscosity-thermal-conductivity coefficient. The function $f(T)$ is a convex strongly nonlinear function satisfying (see [6])

$$
\begin{gathered}
\frac{\partial f}{\partial T}=a(T)>0, \frac{\partial^{2} f}{\partial T^{2}}>\delta>0, \\
\lim _{T \rightarrow+\infty} f(T)=+\infty
\end{gathered}
$$

For example one may choose $f(T)=\left(a T^{2}\right) / 2,(a>0)$ (see [7, p. 1100]). In addition, we encounter the well known cold boundary difficulty, that is, the unburned state is not a stationary point of $(1.1)$ since the "reaction rate function" $\Phi(T) \neq 0$, for $T>0$. One resolution of the cold boundary difficulty can be based on activation energy asymptotics ([10]). However, in our analysis we use the common mathematical idealisation of an ignition temperature, that is, $\Phi$ is modified such that

$$
\Phi(T)= \begin{cases}0 & \text { for } T<T_{i}, \\ \Phi_{1}(T) & \text { for } T \geqslant T_{i},\end{cases}
$$

where $\Phi_{1}(T)$ is a smooth positive function and $T_{i}$ a suitable "ignition temperature" of the reaction. A typical example for $\Phi_{1}(T)$ is the Arrhenius law, $\Phi_{1}(T)$ $=T^{\gamma} e^{-(A / T)}$ for some positive constants $\gamma$ and $A$. Notice that $\Phi(T)$ is discontinuous at the point $T_{i}$. A careful discussion of this assumption and its consequences for detonation and deflegration waves (with one-step chemistry) can be found in [3].

The system (1.1) was proposed by Majda [6] as a model for dynamic combustion, that is, for the interaction between chemical reactions and compressible fluid dynamics. He proved the existence of weak and strong detonations and for $q_{0}$ independent from $T$, assuming a very simple form of $\Phi(T)$. His results are described in terms of liberated energy, $q_{0}$. That is, for fixed $\kappa>0$, he proved that there is a critical liberated energy, $q_{0}^{C R}$, such that when $q_{0}>q_{0}^{C R},(1.1)$ admits a strong detonation combustion profile; when $q_{0}=q_{0}^{C R},(1.1)$ admits a weak detonation combustion profile. Also Rosales and Majda [7] investigated the qualitative model (1.1) in a physical context. Colella, Majda and Roytburd [1] have used fractional step methods based on the use of a second order Godunov method. They demonstrated that the system (1.1) has dynamically stable weak detonations which occur in bifurcating wave patterns from strong detonation initial data. They used system (1.1) both to predict and analyse the theoretical and numerical phenomena. On the other hand, Li [4] studied the system (1.1) when $\beta=0$. He established global existence of the solution to the problem and studied the asymptotic behaviour of the solution. He also proved that the solution converges to a self-sustaining detonation wave and if the data are small, the solution decays to zero like an $N$-wave. Finally, Liu and Ying [5] studied strong detonation waves 
for (1.1) and proved these waves are nonlinearly stable by using an energy method for the fluid variable and a pointwise estimate for the reactant.

Now assume the reaction is exothermic. Then the reaction rate function $\Phi(T)$ has the general form (1.3), and $\beta>0$. Further, consider the natural form where the liberated energy $q_{0}$, depends on $T$, that is, $q_{0}(T)$. We show the existence of traveling waves for Chapman-Jouguet detonation by topological arguments in ordinary differential equations. In addition, the uniqueness of this wave is considered.

The rest of the paper is organised as follows. In Section 2, we introduce the problem and make some related observations. In Section 3,we shall show the existence of traveling wave for Chapman-Jouguet detonation.

\section{ThE hYPOTHESES AND THE PROBLEM}

In this section we first derive a system of ordinary differential equations from (1.1) and then obtain the rest points of the resulting system. A solution $(T(x, \tau), Z(x, \tau))^{T}$ of the system (1.1) is called a traveling wave solution between two states $\left(T_{l}, Z_{l}\right)^{T}$ and $\left(T_{r}, Z_{r}\right)^{T}$, if there is a constant $s \in \mathbb{R}$, which is called the speed of combustion shock wave, satisfying "the jump and entropy conditions"; moreover this solution depends only on the variable $\xi=x-s \tau([8])$. This means that a traveling wave solution of (1.1) has the following form:

$$
(T(x-s \tau), Z(x-s \tau))^{T} .
$$

Thus for a traveling wave solution, the system (1.1) reduces to the system of ordinary differential equations:

$$
\begin{gathered}
-s T_{\xi}+\{f(T)\}_{\xi}-q_{0} Z_{\xi}=\beta T_{\xi \xi}, \\
Z_{\xi}=\kappa \Phi(T) Z .
\end{gathered}
$$

The first equation of (2.1) can be integrated once to give:

$$
\beta T_{\xi}=f(T)-s T-q_{0} Z+C,
$$

where $C$ is the constant of integration. Thus the system (2.1) reduces to the system

$$
\begin{gathered}
\beta T_{\xi}=f(T)-s T-q_{0} Z+C=g(T, Z), \\
Z_{\xi}=\kappa \Phi(T) Z .
\end{gathered}
$$

In order for a solution $u(\xi)=(T(\xi), Z(\xi))^{T}$ to be a traveling wave solution from state $u_{l}=\left(T_{l}, Z_{l}\right)^{T}$ to state $u_{r}=\left(T_{r}, Z_{r}\right)^{T}$, it should satisfy $\lim _{\xi \rightarrow-\infty} u(\xi)=u_{l}$ and 
$\lim _{\xi \rightarrow+\infty} u(\xi)=u_{r}$. Thus $u_{l}$ and $u_{r}$ must be two rest points of the system (2.3). Remembering this, the problem will be investigated in two steps. First, to find all the rest points of the system (2.3) and second, to find all solutions of this system which connect a rest point corresponding to an unburned state to a rest point corresponding to a burned state as $\xi$ increases from $-\infty$ to $+\infty$, whenever $\beta>0$. Now the rest points are solutions of:

$$
\begin{gathered}
f(T)-s T-q_{0} Z+C=0, \\
\kappa \Phi(T) Z=0 .
\end{gathered}
$$

But $\Phi(T)=0$ for $T<T_{i}$, where $T_{i}$ is the ignition temperature, and this set is contained in the region $0<Z \leqslant 1$. Thus from the last equation of (2.4), at a rest point we must have $Z=0$ or $T<T_{i}$.

CASE 1. $Z=0$. From the first equation of (2.4), at a rest point we obtain

$$
g_{0}(T)=g(T, 0)=f(T)-s T+C=0 .
$$

CASE 2. $\Phi(T)=0$. In this case, at the rest point we must have $T<T_{i}$. Then the first equation of (2.4) gives a set of rest points. So by considering the first equation of (2.4) and substituting $Z$ by $m$, we have

$$
g_{m}(T)=g(T, m)=f(T)-s T-q_{0} m+C=0 .
$$

LEMmA 2.1. For all $0 \leqslant m \leqslant 1, g_{m}^{\prime}(T)=0$, for precisely one value of $T$. This is a absolute minimum.

Proof: The lemma follows immediately from

$$
\begin{aligned}
g_{m}^{\prime}(T)=\frac{d g_{m}}{d T} & =f^{\prime}(T)-s=a(T)-s \\
\frac{d^{2} g_{m}}{d T^{2}} & =f^{\prime \prime}(T)>\delta>0 .
\end{aligned}
$$

Lemma 2.2. There is a number $C_{0} \in \mathbb{R}$ such that for $C>C_{0}$, the system (2.5) admits no solutions. For $C=C_{0}$ it admits one solution, and for $C<C_{0}$ it admits two solutions.

Proof: This result follows at once from properties (1.2).

With respect to Lemma 2.2 for $C=C_{0}$, we have only one rest point where $\left(T_{C J}, 0\right)$ represents the Chapman-Jouguet detonation state. In this case, for $m=0$, Lemma 2.1 
along with (1.2) show that the equation (2.5) has exactly one solution $T_{C J}$. Note that the wave speed $s$ satisfies

$$
s=f^{\prime}\left(T_{C J}\right)
$$

Consider now all the critical points of the system (2.3) corresponding to unburned gas states. These points are described by

$$
g\left(T_{m}, m\right)=0, \quad T_{m} \leqslant T_{i},
$$

where $0<m \leqslant 1$ and $T_{i}$ is the ignition temperature which will be obtained later.

By considering the above results the rest points of the system (2.3) are

$$
\begin{aligned}
u_{0} & =\left(T_{C J}, 0\right), \\
u_{m 0} & =\left(T_{m}, m\right), 0<m \leqslant 1, T_{m} \leqslant T_{i},
\end{aligned}
$$

where $T_{i}<T_{C J}$.

In the present work it is assumed that the rest point $u_{0}$, exists.

COROLlaRY 2.3. If the rest point $u_{0}$, exists, then the rest point $u_{m 0}$ exists for some $0<m \leqslant 1$.

A combustion shock wave between $u_{0}$ and $u_{m 0}$ is called a Chapman-Jouguet detonation wave.

From the mathematical point of view, the existence of Chapman-Jouguet detonation wave corresponds to the existence of a complete orbit of the system (2.3) which runs from the rest point $u_{0}$ to $u_{m 0}$ for some $0<m \leqslant 1$. Such an orbit is called a traveling wave solution for the system (1.1).

\section{The existence of Chapman-Jouguet Detonation}

The central theme of this section is the existence of a traveling wave for ChapmanJouguet detonation. In order to show this, we make some observations related to the nature of the unstable manifold of the system (2.3) at the rest point $u_{0}$. Thus we consider the linearised system of (2.3) at the rest point $u_{0}$, which can be written as

$$
\dot{u}=M_{0}\left(u-u_{0}\right)
$$

where

$$
M_{0}=\left[\begin{array}{cc}
\frac{1}{\beta}\left(f^{\prime}\left(T_{C J}\right)-s\right) & -\frac{q_{0}}{\beta} \\
0 & \kappa \Phi\left(T_{C J}\right)
\end{array}\right]
$$


and the entries of the matrix must be considered at the rest point $u_{0}$. Let $h(\lambda)$ be the characteristic polynomial of this matrix. Then we get

$$
h(\lambda)=\left[\frac{1}{\beta}\left(f^{\prime}\left(T_{C J}\right)-s\right)-\lambda\right]\left[\kappa \Phi\left(T_{C J}\right)-\lambda\right] .
$$

From (2.7), we know $f^{\prime}\left(T_{C J}\right)=s$. If $\lambda_{1}$ and $\lambda_{2}$ are the solutions of $h(\lambda)=0$, then at the rest point $u_{0}, \lambda_{1}=0$ and $\lambda_{2}>0$. Thus the following theorem is proved.

THEOREM 3.1. Let $\lambda_{k}, k=1,2$, be the eigenvalues of the matrix $M_{0}$ at the rest point $u_{0}=\left(T_{C J}, 0\right)$. Then at the rest point $u_{0}, \lambda_{1}=0$ and $\lambda_{2}>0$.

We have the following theorem about the eigenvectors at this rest point.

THEOREM 3.2. Let $\left(y_{1}, y_{2}\right)^{T}$ be an eigenvector corresponding to the positive eigenvalue $\lambda_{2}$; then at the rest point $u_{0}$, either $y_{1}>0$ and $y_{2}<0$, or the reverse inequalities hold.

PRoOF: The eigenvector $\left(y_{1}, y_{2}\right)^{T}$ must satisfy the following equation at the rest point $u_{0}$,

$$
-\frac{q_{0}}{\beta} y_{2}=\lambda_{2} y_{1}
$$

and this means that $\operatorname{sgn} y_{2}=-\operatorname{sgn} y_{1}$.

In order to show the existence of a traveling wave, we define:

$$
D=\left\{u \in \mathbb{R}^{2}: 0<Z<1, T<T_{C J}, g(T, Z)<0\right\} .
$$

Now, note that the rest point $u_{0}$, is located on $\partial D$. Moreover, by Theorem 3.1, the unstable manifold at $u_{0}$, is one dimensional. In addition we have the following lemma about this manifold.

Lemma 3.3. Let $D$ be as above, then the unstable manifold at $u_{0}$ intersects $D$ on a curve.

Proof: As we have shown before, the linearised system of (2.3) at the rest point $u_{0}$, has the following form:

$$
\begin{aligned}
\beta \dot{T} & =-q_{0} Z=g_{1 l}(T, Z), \\
\dot{Z} & =\kappa \Phi\left(T_{C J}\right) Z=g_{2 l}(Z) .
\end{aligned}
$$

Let $\left(y_{1}, y_{2}\right)^{T}$ be an eigenvector corresponding to the positive eigenvalue $\lambda_{2}$ $=\kappa \Phi\left(T_{C J}\right)$, at the rest point $u_{0}$. Now consider the solution:

$$
u(\xi)=(T(\xi), Z(\xi))^{T}=\left(y_{1}, y_{2}\right)^{T} e^{\lambda_{2} \xi}+u_{0},
$$


of the linear system (3.2). Then $u(\xi) \in D_{C J}$, where

$$
D_{C J}=\left\{u \in \mathbb{R}^{2}: 0<Z<1\right\} .
$$

To see this notice that

$$
\left(g_{1 \iota}(T, Z), g_{2 \iota}(T, Z)\right)^{T}=M\left(u-u_{0}\right)=M Y e^{\lambda_{2} \xi}=\lambda_{2} Y e^{\lambda_{2} \xi}=\left(\lambda_{2} y_{1}, \lambda_{2} y_{2}\right)^{T} e^{\lambda_{2} \xi} .
$$

By Theorem 3.2, we may assume that $y_{1}<0$ and $y_{2}>0$. Since $\lambda_{2}>0$, it follows from the last equality that $\left(g_{1 l}(T, Z), g_{2 l}(T, Z)\right) \in D_{C J}$. This means that the unstable manifold of (3.2), at the rest point $u_{0}$, which is just the line

$$
M_{C J}=\left\{u \in \mathbb{R}^{2}: u-u_{0}=\left(y_{1}, y_{2}\right)^{T} s, s \in \mathbb{R}\right\},
$$

lies in $D_{C J}$ for $s>0$, and lies in $D$ for $s>0$ and small. Thus the unstable manifold of the system (3.2), at the rest point $u_{0}$ intersects $D$ on a curve.

Now consider the following system of ordinary differential equations:

$$
\begin{aligned}
\beta \dot{T} & =f(T)-s T-q_{0} Z+C=g(T, Z), \\
\dot{Z} & =\kappa \Phi_{1}(T) Z:=g_{3}(T, Z)
\end{aligned}
$$

where $\Phi_{1}(T)$ is defined by (1.3). Notice that the above system leads us to a proof of the existence of a traveling wave for the Chapman-Jouguet detonation wave. The proof of the next lemma is similar to the proof of [2, Theorem 3.1].

Lemma 3.4. Let $D$ be as above. Then there is a unique orbit of the system (3.3) which lies in $D$, its $\alpha$-limit set is $u_{0}$, and this orbit intersects the set $\Delta=\{u$ $\in \bar{D}: g(T, Z)<0, T<T_{C J}$, and $\left.Z=1\right\}$. Along this orbit, $T(\xi)$ is decreasing and $Z(\xi)$ is increasing.

Proof: The proof is organised in the following steps.

STEP 1. The system (3.3) is gradient like with respect to $h(u)=Z$ in $D$, and is locally Lipschitz in a neighbourhood of $\bar{D}$.

STEP 2. Note that $D$ is homeomorphic to the parabola $\left\{X \in \mathbb{R}^{2}: x_{1}^{2}<x_{2}, 0<x_{2}<1\right\}$ and so $\{u \in \bar{D}: h(u)=c\}$ corresponds to the set $\left\{X \in \mathbb{R}^{2}: x_{1}^{2}<x_{2}, x_{2}=c\right\}$ for $c \in[0,1]$ under the homeomorphism.

STEP 3. The $u_{0}$ is the only rest point of (3.3) which is in $\{u \in \bar{D}: h(u)=0\}$.

STEP 4. Let $E=\{u \in \partial D: h(u)<1\}$. For $p \in \bar{E}-\left\{u_{0}\right\}, p . \xi \in D$ for small positive $\xi$ and $p . \xi \notin \partial D$ for small $|\xi| \neq 0$. To see this, let $u^{\prime} \in\{u \in \partial D: 0<Z<1\}$. Then 
$g\left(u^{\prime}\right)=0$ or $T=T_{C J}$. Now, suppose $g\left(u^{\prime}\right)=0$. If we differentiate $g(T, Z)$ along the orbits of (3.3) we obtain:

$$
\frac{d g(u)}{d \xi}=\left(\frac{\partial f(T)}{\partial T}-s-\left(q_{0}\right)_{T} Z\right) \dot{T}-q_{0} \dot{Z}
$$

Hence

$$
\left.\frac{d g(u)}{d \xi}\right|_{I_{g\left(u^{\prime}\right)=0}}=\left(\left(\frac{\partial f(T)}{\partial T}-s-\left(q_{0}\right)_{T} Z\right) \frac{g(u)}{\beta}-q_{0} \dot{Z}\right)_{I_{g\left(u^{\prime}\right)=0}}=-q_{0} \dot{Z}<0,
$$

and

$$
\frac{d T}{d \xi}_{\left.\right|_{T=T_{C J}}}=\frac{1}{\beta}\left(f(T)-s T-q_{0} Z+C\right)_{\left.\right|_{T=T_{C J}}}=\frac{1}{\beta} g\left(T_{C J}, Z\right)<0 .
$$

Thus the flow come in $\bar{D}$ on $g\left(u^{\prime}\right)=0$ and $T=T_{C J}$.

STEP 5. Let $F=\partial D-\bar{E}=\{u \in \partial D: Z=1\}$. For $p \in F, p . t \notin D$ for $\xi>0$ and small. To see this, differentiate $Z$ along the orbits of (3.3) we obtain

$$
\frac{d Z}{d \xi}_{\mid Z=1}=\kappa \Phi(T) Z_{\mid z=1}>0,
$$

Thus the flow goes out of $\bar{D}$ on $Z=1$.

STEP 6. From the Steps 1-5, there is a point $p \in F$ such that $p . \xi \in D$ for $\xi<0$ and $\lim _{\xi \rightarrow-\infty} p . \xi=u_{0}$.

Proof OF SteP 6: Suppose such a point $p$ does not exist. Then for each $y \in F$ there is a $\xi<0$ such that $y . \xi \notin \bar{D}$. Then, by Step 4 , there is $\xi(y)<0$ such that $y .(\xi(y), 0) \subset D, y . \xi(y) \in \bar{E}$ and $y .\left(\xi(y)-\varepsilon_{1}\right) \notin \bar{D}$ for some $\varepsilon_{1}>0$. Now define $\varphi: \bar{F} \rightarrow \bar{E}$, by $\varphi(y)=y \cdot \xi(y)$. It follows, from continuity of $y . \xi$ with respect to the initial condition $y$, that $\xi(y)$ and $\phi(y)$ are continuous. Now we shall show that $\varphi: \bar{F} \rightarrow \varphi(\bar{F})$ is a homeomorphism. By definition, $\varphi$ is one to one. So it suffices to show that, if $V$ is open in $\bar{F}$ then $\varphi(V)$ is open in $\varphi(\bar{F})$. If $V$ is open in $\bar{F}$, then $\bar{F} \backslash V$ is compact in $\bar{F}$. Hence $\varphi(\bar{F} \backslash V)$ is closed. Thus $\varphi(V)$ is open in $\varphi(\bar{F})$.

Now we show that $\varphi: \bar{F} \rightarrow \bar{E}$ is onto. To see this, note that $\varphi(\partial F)=\partial E$ and $\varphi(F)=\operatorname{int} \bar{E}$, where int $\bar{E}$ means interior of $\bar{E}$ with respect to $\partial D$. Since $F$ is open in $\partial D$, by the Brouwer Theorem on the Invariance of Domain $([9]), \varphi(F)$ must be open in $\partial D$. Thus it must be open in int $\bar{E}$ with respect to $\partial D$. On the other hand, we have $\varphi(F)=\varphi(\bar{F}) \cap \operatorname{int} \bar{E}$ and $\varphi(\bar{F})$ is closed in $\bar{E}$. Hence $\varphi(F)$ must be closed in int $\bar{E}$. Since $\varphi(F) \neq \phi$, it follows that $\varphi(F)=\operatorname{int} \bar{E}$. Also for $y \in \partial F$, we have $\varphi(y)=y$. It then follows that $\varphi: \bar{F} \rightarrow \bar{E}$ is onto. This means that there is a point $y_{0} \in F$ with 
$\xi\left(y_{0}\right)<0$ such that $y_{0} . \xi\left(y_{0}\right)=u_{0}$. This is impossible as $u_{0}$ is a rest point of (3.3). Therefore there is a point $p \in D$ such that $p . \xi$ is defined for all $\xi \leqslant 0$ and lying in $D$ for $\xi<0$. The $\alpha$-limit set of $p$. $\xi$ must be $u_{0}$, as the flow is gradient-like and $u_{0}$ is the only rest point of $(3.3)$ in $\bar{D}$. This completes the proof of the claim.

Thus by the last step, there must be an orbit of the system (3.3) lying in D, starting at a point on the surface $Z=1$ and running to the point $u_{0}$ as $\xi \rightarrow-\infty$. Finally from the system (3.3) and the set $D$ it follows that along this orbit $Z(\xi)$ and $T(\xi)$ are increasing and decreasing respectively.

Let $\widetilde{u}(\xi), \xi \in\left(-\infty, \xi_{0}\right]$ be the orbit which is given by the above lemma. Then $\tilde{u}\left(\xi_{0}\right) \in\{u \in \bar{D}: Z=1\}$ and $\lim _{\xi \rightarrow-\infty} \tilde{u}(\xi)=u_{0}$. We have the following lemma about the orbit $\widetilde{u}(\xi)$.

LEMMA 3.5. Let $\tilde{u}(\xi)$ be as above. Then there is $0<\tilde{Z} \leqslant 1$, such that for $\kappa \beta$ small enough, the orbit $\widetilde{u}(\xi)$ meets the line $T=T_{i}$, at $\widetilde{u}(\widetilde{\xi})=\left(T_{i}, \widetilde{Z}\right)$, for some $\tilde{\xi} \in\left(-\infty, \xi_{0}\right)$.

Proof: Let $u_{0}$ be as (2.8). Choose the line $P: T-T_{C J}=0$, where $T_{C J}$ is the first component of $u_{0}$. Let $\left(T_{i}, Z_{i}\right)^{T}$ be the unique solution of the equation

$$
g(u)=0, T=T_{i}
$$

Then we obtain $Z_{i}=\left(1 / q_{0}\right)\left(f\left(T_{i}\right)-s T_{i}+C\right)$. Also from $T_{m}<T_{i}<T_{C J}$, it follows that $0<Z_{i}<1$, and $\left\{u \in \bar{D}: g(u)=0, Z_{i}<Z<1\right\} \subset\left\{u \in \bar{D}: T \leqslant T_{i}\right\}$. Now consider the line $P^{\prime}: T-T_{i}=0$. Since $u_{m 0} \in\left\{u \in \bar{D}:\left(T-T_{i}\right)<0\right\}$, we can choose $Z_{i}<Z_{0}<1$ such that

$$
\left\{u \in \bar{D}: g(u)=0, Z_{0}<Z<1\right\} \subset\left\{u \in \bar{D}: T-T_{i}<0\right\} .
$$

Let $D_{0}=\left\{u \in D: T_{i}<T<T_{C J}, Z_{0}<Z<1\right\}$ and $\delta=\max _{u \in \bar{D}_{0}} g(u)$. Then $\delta<0$.

Now suppose the orbit $\widetilde{u}(\xi), \xi \in\left(-\infty, \xi_{0}\right]$, does not meet the set $\{u \in D: T$ $\left.=T_{i}, 0<Z \leqslant 1\right\}$. Let $\xi_{1}<\xi_{0}$ be the solution of the equation $\tilde{Z}(\xi)=Z_{0}$, where $\tilde{Z}(\xi)$ is the second component of $\widetilde{u}(\xi)$. Since $d T / d \xi=(1 / \beta) g(u)<0, T$ is decreasing along the orbit $\tilde{u}(\xi)$, and it follows that $\tilde{u}(\xi)$ remains in $D_{0}$ for $\xi_{1}<\xi<\xi_{0}$. Now along the orbit $\widetilde{u}(\xi)$ in $D_{0}$ we must have:

$$
\begin{aligned}
-\frac{d T}{d Z} & =\frac{1}{(d Z) /(d \xi)}\left(-\frac{d T}{d \xi}\right) \\
& =\frac{1}{\kappa Z \Phi_{1}(T)}\left(\frac{-1}{\beta} g(u)\right) \\
& \geqslant \frac{\sigma(-\delta)}{\kappa \beta}>0
\end{aligned}
$$


where $1 / \sigma=\max _{u \in \bar{D}} \Phi_{1}(T) Z$. Let $T_{0}=T\left(\xi_{0}\right)$. Then $T_{i}<T_{0}$, if $\tilde{u}(\xi)$ does not meet $T_{i}$. Therefore

$$
\begin{aligned}
T_{C J}-T_{i} \geqslant T_{C J}-T_{0} & =-\int_{-\infty}^{\xi_{0}} \frac{1}{\beta} g(u) d \xi \\
& =\int_{-\infty}^{\xi_{0}} \frac{1}{\beta}(-g(u)) d \xi \\
& \geqslant \int_{\xi_{1}}^{\xi_{0}} \frac{1}{\beta}(-g(u)) d \xi \\
& =\int_{z_{0}}^{1} \frac{1}{\kappa Z \Phi_{1}(T)}\left(\frac{-1}{\beta} g(u)\right) d Z \\
& \geqslant \frac{\sigma(-\delta)}{\kappa \beta}\left(1-Z_{0}\right),
\end{aligned}
$$

which is impossible for $\kappa \beta$ small enough. Thus there is a $\tilde{\xi} \in\left(-\infty, \xi_{0}\right)$ such that the orbit $\widetilde{u}(\xi)$ meets the line $T=T_{i}$ at the point $\widetilde{u}_{i}=\left(\widetilde{T}_{i}, \widetilde{Z}_{i}\right)^{T}$, where $\widetilde{T}_{i}=T_{i}$, and $\widetilde{Z}_{i}=\widetilde{Z}(\widetilde{\xi})$.

From now on we assume that $\kappa \beta$ is small enough, or the orbit $\widetilde{u}(\xi)$ meets the line $T=T_{i}$ at the point $\widetilde{u}_{i}=\left(\widetilde{T}_{i}, \widetilde{Z}_{i}\right)^{T}$, where $\widetilde{T}_{i}=T_{i}$, and $\widetilde{Z}_{i}=\widetilde{Z}(\widetilde{\xi})$. We call the point $\tilde{u}_{i}$ the ignition point. According to Lemma 3.4, this point for Chapman-Jouguet detonation is unique.

Now we have our main theorem as follows.

THEOREM 3.6. Suppose that the system (2.3) admits the rest points $u_{0}$ and $u_{m 0}$, for some $0<m \leqslant 1$. If $\kappa \beta$ is small enough, then there is a unique orbit of the system (2.3) which runs from $u_{0}$ to $u_{m 0}$, for some $0<m \leqslant 1$.

Proof: In the region $T<T_{i}$, the last equation of (2.3) becomes $\dot{Z}=0$. Thus, in this region, along the orbits of this system $Z(\xi)$ is constant. Here we let $Z(\xi)=\widetilde{Z}_{i}$, where $\widetilde{Z}_{i}$ is the second component of $\widetilde{u}_{i}$, (the ignition point). On the surface $Z=\widetilde{Z}_{i}$, the system (2.3) reduces to the following one dimensional system of equations in the region $T \leqslant T_{i}$ :

$$
\beta \dot{T}=f(T)-s T-q_{0} \widetilde{Z}_{i}+C:=F(T) .
$$

Now consider the region $D^{\prime}=\left\{T \in \mathbb{R}: F(T)<0, T<T_{i}\right\}$. Notice that $T=T_{i} \in \partial D^{\prime}$. Also it is trivial to see that any orbit of (3.4) initiating at a point on $\partial D^{\prime} \cap\left\{T: T=T_{i}\right\}$ approaches the unique rest point of the system (3.4) which is located in the region $T<T_{i}$, as $\xi$ tends to $+\infty$. We denote this rest point by $\overline{T_{i}}$. 
Again consider the ignition point $\widetilde{u_{i}}=\left(T_{i}, \widetilde{Z_{i}}\right)$. By the above argument, there is a unique orbit of the system $(2.3)$, say $\widetilde{u}(\xi)=(\tilde{\widetilde{T}}(\xi), \widetilde{Z}(\xi)), \tilde{\xi}<\xi<+\infty$, with

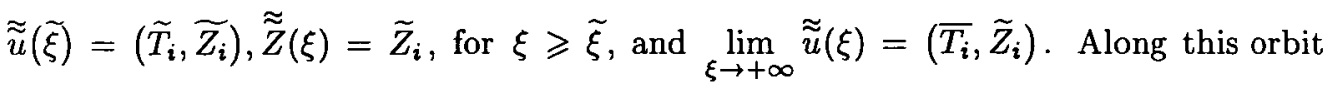
$T(\xi)$ is increasing and $Z(\xi)$ is constant. This orbit lies in $D$, the domain which is used in the proof of Lemma 3.4.

Now define,

$$
u(\xi)= \begin{cases}\widetilde{u}(\xi) & \xi<\tilde{\xi} \\ \widetilde{u}(\xi) & \xi \geqslant \widetilde{\xi}\end{cases}
$$

Then $u(\xi)$ is a complete orbit of the system (2.3) lying in $D$ and runs from $u_{0}$ to $u_{m 0}$ for some $0<m \leqslant 1$. This completes the proof.

\section{REFERENCES}

[1] P. Colella, A. Majda and V. Roytburd, 'Theoretical and structure for reacting shock waves', SIAM J. Sci. Statist. Comput. 7 (1986), 1059-1080.

[2] M. Hesaaraki and A. Razani, 'On the existence of Chapman-Jouguet detonation wave', Bull. Austral. Math. Soc. 63 (2001), 485-496.

[3] A.K. Kapila, B.J. Matkowsky and A. Van Harten, 'An asymptotic theory of deflagration and detonations 1. The steady solutions', SIAM J. Appl. Math. 43 (1983), 491-519.

[4] T. Li, 'On the initiation problem for a combustion model', J. Differential Equations 112 (1994), 351-373.

[5] T.P. Liu and L.A. Ying, 'Nonlinear stability of strong detonation for a viscous combustion model', SIAM J. Math. Anal. 26 (1995), 519-528.

[6] A. Majda, 'A qualitative model for dynamic combustion', SIAM J. Appl. Math. 41 (1981), 70-93.

[7] R. Rosales and A. Majda, 'Weakly nonlinear detonation waves', SIAM J. Appl. Math. 43 (1983), 1086-1118.

[8] J.A. Smoller, Shock waves and reacting diffusion equations (Springer-Verlag, New York, Heidelberg, Berlin, 1994).

[9] J.W. Vick, Homology theory, Pure and Applied Math. 53 (Academic Press Inc., New York, London, 1970).

[10] F.A. Williams, Combustion theory (Benjamin/Cummings, Melon Park, CA, 1985).

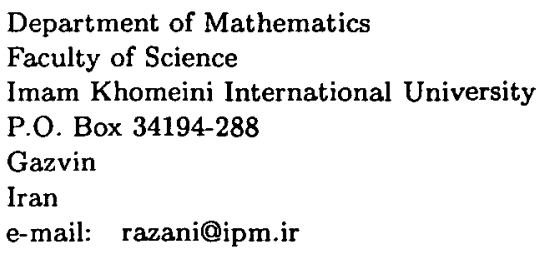

